\title{
Precise Point Positioning in the Airborne Mode
}

\begin{abstract}
Ahmed El-Mowafy ${ }^{*}$
Abstract: The Global Positioning System (GPS) is widely used for positioning in the airborne mode, such as in navigation as a supplementary system and for geo-referencing of cameras in mapping and surveillance by aircrafts and Unmanned Aerial Vehicles (UAV). For real-time applications, the traditional differential positioning method cannot usually be used due to its need for data from a ground reference station that should be located within a short range. However, the Precise Point Positioning (PPP) approach can provide an attractive alternative that is based on processing of un-differenced observations from a single GPS receiver. It employs precise satellite orbits and satellite clock corrections. These data are freely available via the internet from several sources, e.g. the International GNSS Service. This data can also broadcast from satellites, such as the new Japanese satellite system QZSS. The PPP can achieve positioning precision and accuracy at the sub-decimetre level.

In this paper, the functional and stochastic mathematical modelling used in PPP is discussed. Results of applying the PPP method in an airborne test using a small fixed-wing aircraft are presented. Although the data were processed in a post-mission mode, the same technique can be applied in real time, if precise orbits and clock corrections are available. To evolute the performance of the PPP approach, a reference trajectory was established by differential positioning of the same GPS observations with data from a ground reference station. The coordinate results from the two approaches, PPP and differential, were compared and statistically evaluated. For the test at hand, positioning accuracy at the $\mathrm{cm}$-to-decimetre was achieved for easting and northing coordinates, and doubles that value for height estimation.
\end{abstract}

Keywords: Precise Point Positioning, GPS, aviation, mapping

\section{Introduction}

The use of the global navigation satellite systems (GNSS) for navigation in civil aviation is expanding. It is estimated that from 2015, most new commercial aircraft will be fitted with a dual GPS/Galileo system to enhance precise navigation and make it safer, [1]. At the moment, GPS is approved as a stand-alone aid for non-precision approaches [2], e.g. as a supplementary navigation system, and for positioning of non safety-of-life applications such as aerial mapping. Due to the presence of satellite, media and receiver errors, a GPS system in a standalone mode can generally produce positioning with accuracy from 1 to $10 \mathrm{~m}$. Therefore, some techniques are required to eliminate or reduce measurement errors in order to use the system for applications demanding better accuracy, such as aerial mapping in postmission processing, or aviation in real-time. For instance, in CAT-I phase of aviation, which includes enroute flying, positioning by GPS is dependent on receiving corrections from Satellite Based Augmentation Systems (SBAS), such that a positioning accuracy at the range 1-3 m can be achieved. However, in CAT-III, which is the more demanding phase in aviation

\footnotetext{
*PhD., Senior Lecturer, Curtin University, GPO Box U1987, Perth, WA 6845, Australia

Email: a.el-mowafy@ curtin.edu.au
} 
as the aircraft gets into the precise final approach and landing, accuracy better than $0.6 \mathrm{~m}$ is required in estimation of the height and $3 \mathrm{~m}$ in horizontal coordinates. It is proven with the continuous development of real-time single or network reference systems that with a good set of phase-measurement corrections, such required accuracy and precision can be met, [3].

Traditionally, GPS positioning errors are reduced by double differencing of the rover measurements with observations from a reference station of known coordinates, making centimetre-accuracy positioning possible. The shortcoming of this approach is the need to use synchronous measurements at the reference and the rover, and that the reference-to-rover distance should typically be less than $30 \mathrm{~km}$. Currently, in the airborne mode, these two conditions are hard to achieve all the time, particularly for aviation, as firstly, it is expensive to send the reference station data in real-time to the aircraft, and technically, it is hard to maintain receiving the corrections without experiencing some breaks. Secondly, finding a reference station that is located within a reasonable range might be difficult for cases such as emergency landing or rescue operations.

An alternative method of processing GNSS measurements is the precise point positioning (PPP) approach, which is based on the processing of un-differenced observations from a single GPS receiver, and employing precise satellite orbits and clock corrections. Therefore, no reference stations are needed. However, to reach sub-decimetre positioning accuracy, the phase measurements should be used, and thus, their ambiguities should be determined. Typically, they are computed as float ambiguities with real-values along with the positioning unknowns, [4]. As a result, a long time is needed, typically 10-30 minutes, for the solution to converge before a reliable estimate of the float ambiguities can be determined, [5]. In this paper, a brief presentation of the PPP method and its application in the airborne mode is given. Results of an airborne test using PPP for positioning of a fixed-wing aircraft will be presented and discussed.

\section{Functional Modelling of the PPP Method}

The code and phase observation equations of the GPS can be formulated in metre units as follows:

$$
\begin{aligned}
& \rho_{(t)}=r_{(t, t-\tau)}+d s_{(t-\tau)}+c d T_{(t)}-c d t_{(t-\tau)}+d_{\text {iono }}+d_{\text {tropo }}+\varepsilon(\rho) \\
& \phi_{(t)}=r_{(t, t-\tau)}+d s_{(t-\tau)}+c d T_{(t)}-c d t_{(t-\tau)}-d_{\text {iono }}+d_{\text {tropo }}+\lambda N+\varepsilon(\phi)
\end{aligned}
$$

where $\rho_{(t)}$ and $\phi_{(t)}$ are the pseudo-range code and the phase measurements, respectively, at the time of receiving the data $(t),(t-\tau)$ denotes the satellite time, where $\tau$ refers to the travel time between the satellite and the receiver. $r_{(t, t-\tau)}$ is the true geometric range, $d s$ is the orbital error, c denotes the speed of light, $d T$ and $d t$ are the receiver and satellite clock errors, $d_{\text {iono }}$ and $d_{\text {tropo }}$ are the ionosphere and troposphere delays, respectively. $\lambda$ denotes the carrier-phase wavelength, $N$ is the integer phase ambiguity, and $\varepsilon$ denotes the measurement noise, including multipath and the antenna phase centre variation.

To reduce orbital errors in the PPP approach, the user needs to utilize precise orbits. In addition, due to the fact that GPS satellite clocks are somehow unstable and difficult to be modelled; corrections to satellite clocks are used. The precise orbits and clock corrections can be obtained from the International GNSS Service (IGS) for free via the internet. Table 1 gives an overview of IGS currently available satellite products, which shows the accuracy, expected 
latency in updating these products, frequency of updates, and sample interval. In general, best results can be obtained using the Final IGS products; however, this is only applicable for postprocessing applications. For real-time applications, a communication link is needed to download the products, and under the Real Time (RT)-IGS pilot project, 'near' real-time products are currently being developed with an accuracy and sampling interval that give the user the possibility to obtain results near the results obtained with Rapid IGS products. For the user that has no real-time communication link, the Predicted IGS orbits are the best option available; however, they give the least accuracy. A few organizations are currently developing real-time products, such as the REal-Time system for CLock Estimation (RETICLE) by the German Space Operations Centre of DLR, and Natural Resources Canada (NRCan).

Table 1 Precise orbits and clock corrections available

\begin{tabular}{|c|c|c|c|c|c|}
\hline \multicolumn{2}{|c|}{$\begin{array}{c}\text { GPS Satellite Ephemerides/ } \\
\text { Satellite Clocks }\end{array}$} & Accuracy & Latency & Updates & $\begin{array}{l}\text { Sample } \\
\text { Interval }\end{array}$ \\
\hline \multirow{2}{*}{ Broadcast } & orbits & $\sim 100 \mathrm{~cm}$ & \multirow{2}{*}{ real time } & \multirow{2}{*}{ daily } & \multirow{2}{*}{2 hours } \\
\hline & satellite clocks & $\sim 2.5 \mathrm{~ns}$ & & & \\
\hline \multirow{2}{*}{ Ultra Rapid (predicted half) } & orbits & $\sim 5 \mathrm{~cm}$ & \multirow{2}{*}{ real time } & \multirow{2}{*}{ at $03,09,15,21 \mathrm{UTC}$} & \multirow{2}{*}{$15 \mathrm{~min}$} \\
\hline & satellite clocks & $\sim 1.5 \mathrm{~ns}$ & & & \\
\hline \multirow{2}{*}{ Ultra Rapid (observed half) } & orbits & $\sim 3 \mathrm{~cm}$ & \multirow{2}{*}{$3-9$ hours } & \multirow{2}{*}{ at $03,09,15,21 \mathrm{UTC}$} & \multirow{2}{*}{$15 \mathrm{~min}$} \\
\hline & satellite clocks & $\sim 50 \mathrm{ps}$ & & & \\
\hline \multirow{2}{*}{ Rapid } & orbits & $\sim 2.5 \mathrm{~cm}$ & \multirow{2}{*}{$17-41$ hours } & \multirow{2}{*}{ at 17 UTC daily } & $15 \mathrm{~min}$ \\
\hline & satellite clocks & $\sim 25 \mathrm{ps}$ & & & $5 \mathrm{~min}$ \\
\hline \multirow{2}{*}{ Final } & orbits & $\sim 2.5 \mathrm{~cm}$ & \multirow{2}{*}{$12-18$ days } & \multirow{2}{*}{ every Thursday } & $15 \mathrm{~min}$ \\
\hline & satellite clocks & $\sim 20 \mathrm{ps}$ & & & $5 \mathrm{~min} \& 30 \mathrm{sec}$ \\
\hline \multirow{2}{*}{$\begin{array}{c}\text { DLR } \\
\text { RETICLE }\end{array}$} & orbits & $\sim 2.5 \mathrm{~cm}$ & \multirow{2}{*}{$5 \mathrm{sec}$} & \multirow{2}{*}{$10 \mathrm{sec}$} & \multirow{2}{*}{$10 \mathrm{sec} \& 30 \mathrm{sec}$} \\
\hline & satellite clocks & $\sim 0.5 \mathrm{~ns}$ & & & \\
\hline
\end{tabular}

The ionosphere can be considered the dominant source of error in GPS positioning. Since the ionosphere is dispersive, i.e. frequency dependent, the most common method of PPP is to use a combination of dual frequency observations to eliminate the first-order ionospheric error, which accounts for more than $99 \%$ of this error. In addition, to minimize the impact of noise from code measurements, a code-phase combination in the form of their average can be used for the two frequencies, [6]. This significantly helps in convergence of the PPP solution, particularly while solving for the ambiguities. A traditional phase combination can also be used. Thus, the model equations can be given as:

$$
\begin{aligned}
\rho_{L i(t)}^{I F}= & \frac{P(L i)+\phi(L i)}{2}=r_{(t, t-\tau)}+d s_{(t-\tau)}+c d T_{(t)}-c d t_{(t-\tau)}+d_{t r o p o}+0.5 \lambda_{i} N_{i}+0.5 \\
& (\varepsilon(\rho(L i)+\phi(L i)) \\
\phi_{(t)}^{I F}= & \frac{f_{1}^{2} \cdot \phi_{1}-f_{2}^{2} \cdot \phi_{2}}{f_{1}^{2}-f_{2}^{2}}=r_{(t, t-\tau)}+d s_{(t-\tau)}+c d T_{(t)}-c d t_{(t-\tau)}+d_{t r o p o}+\frac{c \cdot f_{1} \cdot N_{1}-c \cdot f_{2} \cdot N_{2}}{f_{1}^{2}-f_{2}^{2}}+\varepsilon\left(\phi^{I F}\right)
\end{aligned}
$$

where $I F$ denotes the ionosphere free operator, $f_{1}$ and $f_{2}$ are the frequencies of the carrier waves $L_{1}$ and $L_{2}$, respectively, $\mathrm{i}$ is the frequency operator, where $\mathrm{i}=1$ for $\mathrm{L} 1$ and $\mathrm{i}=2$ for $\mathrm{L} 2$. The ionosphere has the same value for the code and phase measurements but with the opposite sign. Thus, one can see that, their sum eliminates the ionosphere error in Eq.(3). The use of Eq. (3) reduces the code noise, and thus, after collecting a long data set, helps in estimating near-fixed float ambiguities in Eq. (4) if the value of the remaining errors is small enough.

On the other hand, the total tropospheric range delay resulting from propagation of the satellite signals through the neutral atmosphere is expressed as the sum of the hydrostatic and 
wet components. Range delays resulting from the hydrostatic component of the (dry) troposphere, which is the major part of tropospheric error, can be computed with an accuracy of a few millimetres using empirical models (e.g. Saastamionen, Hopfield, etc.). In addition, a good mapping function is required to project the satellite-to-user troposphere delay into the zenith direction. The wet part of the troposphere (approximately $2-10 \%$ of the total) is hard to model as it needs wet vapour measurements, which are usually not available to normal users. Thus, in PPP, if detailed values of the wet troposphere zenith delay are not available from a local network, this error can be taken as an additional unknown.

The estimation of un-differenced ambiguities in PPP is carried out using an on-the-fly approach in the standalone mode. However, a non-zero initial phase is experienced due to the un-synchronization of the satellite-transmitted and receiver-generated signals, which is usually cancelled out in the traditional double-differencing approach. As a result, the ambiguities in PPP are real numbers, [8]. Thus, integer un-differenced ambiguities are first estimated as constants. Next, they are adjusted to their float numbers as the data accumulate and the filter converges to a stable condition. This convergence process usually takes several minutes leading to one of the main limitations of PPP.

From the above, the vector of unknowns typically includes the three position coordinate parameters $(x, y, z)_{u}$, a receiver clock offset parameter, the wet tropospheric component $\left(d_{w e t}\right)$, and the float ambiguities. The state vector can be formulated as, [7]:

$$
\bar{X}_{u}=\left[(x, y, z)_{u}, c d T_{(t)}, d_{w e t},(N)_{1 \ldots n}\right]^{T}
$$

Where $\mathrm{n}$ is the number of observed satellites. In PPP, processing of un-differenced measurements is usually carried out by using Kalman filtering. One however should note that since positioning is performed without differencing, some errors that are mostly eliminated in the differential approach have to be modelled out in the PPP approach. These errors are basically attributed to physical phenomena, and include:

- relativity error, which is a function of the satellite motion and the Earth's gravity;

- Sagnac delay caused by the Earth's rotation during the transition time of the satellite signal;

- phase wind up, due to the relative motion and rotation of the satellite and receiver;

- inter-frequency bias, resulting if using L1 or L2 alone.

Some information about the definition and modelling of these errors are given in [9, 10]. In the airborne mode, errors such as earth tide and ocean tide loading, which are considered for land applications, are ignored.

\section{Stochastic Modelling in PPP}

Obtaining a consistent stochastic model is important in the estimation process. However, such a task is still a challenge. If the stochastic model is incorrect, poor results can be obtained. In addition, an incorrect stochastic model makes it difficult to estimate good float ambiguities, and accordingly inaccurate positioning may result. A good stochastic modelling should involve representation of stochastic behaviour of single measurement errors, their possible cross-correlation, if exist, and their temporal correlation. Thus, such stochastic model should incorporate a fully populated covariance matrix. An example of stochastic modelling used in PPP is given in Table 2, [11]. 
Table 2 Example of Stochastic modeling in PPP

\begin{tabular}{c|c|c}
\hline Component & Stochastic model & $\begin{array}{c}\text { Assumed initial stochastic } \\
\text { parameters }\end{array}$ \\
\hline Measurements & $\begin{array}{c}\text { Uncorrelated individual code and } \\
\text { phase measurements. } \\
\text { Correlated in the observation } \\
\text { equations by error propagation }\end{array}$ & $\begin{array}{c}\text { weight is a function of satellite } \\
\text { elevation angle }\end{array}$ \\
\hline $\begin{array}{c}\text { Position } \\
\text { Receiver } \\
\text { clock error }\end{array}$ & random-walk process & $\begin{array}{l}\text { spectral density }=0-770 \mathrm{~m}^{2} / \mathrm{s} \\
\text { for static and moving on land, } \\
\text { spectral density }=10^{5} \mathrm{~m}^{2} / \mathrm{s} \mathrm{for} \\
\text { marine and airborne modes }\end{array}$ \\
\hline $\begin{array}{c}\text { troposphere } \\
\text { manbiguities }\end{array}$ & first order Gauss-Markov process & $\begin{array}{c}\text { correlation time can be taken } \\
\text { as several minutes. } \\
\text { Spectral density }=8 \times 10^{-12} \mathrm{~m}^{2} / \mathrm{s}\end{array}$ \\
\hline $\begin{array}{c}\text { Initial phase } \\
\text { biases }\end{array}$ & Constant & - \\
\hline
\end{tabular}

\section{Some Aspects in Using PPP in the Airborne Mode}

Applying PPP in post-mission data processing can without difficulty be performed in the airborne mode due to the fact that obtaining precise orbits, satellite clock corrections, and atmospheric corrections when available, can easily be carried out. Thus, PPP can be implemented in aerial mapping and post-mission surveillance. Recall that the main limitation in applying PPP is the long time needed to estimate good real values for the ambiguities. However, this should not be a problem if data longer than 20-30 minutes are collected, which is the period needed to achieve this determination. While positioning can be carried out as usual after determination of the ambiguities, a position solution accurate to the sub-decimetre level can be obtained for the period taken for ambiguity determination by backward processing of data.

For real-time applications, such as aviation and real-time surveillance, obtaining the precise orbits, satellite clock corrections or atmospheric corrections can represent a problem. Technically, this information can be received through the internet via satellite communication. However, this is an expensive option. The cheaper option, which represents the future of PPP, is to get the precise orbits and satellite clock corrections through dedicated free-to-air satellite signals such as the LEX signal of the Japanese system Quasi-Zenith Satellite System (QZSS). Currently, this signal is in the technical/application verification phase and will be in the standard operational phase in mid 2011. 


\section{Testing PPP in the Airborne Mode}

To evaluate the performance of PPP in the airborne mode, data from an airborne test conducted in Delft, Holland on $1^{\text {st }}$ November, 2007, were processed and analysed. A Septentrio PolarRx2 dual-frequency receiver was used for GPS data collection, where the antenna was mounted on the cockpit of a small fixed-wing aircraft. The flight period was from 10:06:15 to 14:00:00, thus, processing of almost 4 hours of kinematic data. The original data was recorded at a sampling interval of $10 \mathrm{~Hz}$. For comparison reasons, as will be explained later, the data were decimated to $1 \mathrm{~Hz}$ intervals.

The airborne data were first post-processed using the PPP approach online using the service provided by the Geodetic Survey Division (GSD) of Natural Resources Canada known as the Canadian Spatial Reference System PPP (CSRS-PPP). This service was designed to be, as much as possible, a self-serve application, [10]. It can be used to process GPS observations made anywhere on or near Earth in static or kinematic modes, at any time of day. In our test, the positions are presented in the International Terrestrial Reference Frame (ITRF), currently ITRF2005, [12]. Since the data were collected in 2007, PPP processing used the precise IGS orbits. The processing also included cycle-slip filtering, applying satellite and receiver antenna phase centres offsets, and a reference frame transformation. The statistical information computed from PPP processing such as standard deviations will be shown to present precision of the method.

For accuracy assessment, the same GPS data of the aircraft receiver were processed in a differential mode with data from a ground reference station, collected at $1 \mathrm{~Hz}$ sampling rate. The PPP solution was compared with the solution of the differential positioning, where the latter was taken as the reference for comparison. This is due to the fact that differential positioning is a well established technique that can give $\mathrm{mm}$ to $\mathrm{cm}$ positioning accuracy as the ambiguities are fixed to their integer values and most errors are reduced or cancelled. The reference station was selected almost at the middle of the course to have the shortest distance possible to the terminal points, which are the most critical, range-wise. Figure 1 shows the trajectory of flight phase of the test and the reference station location.

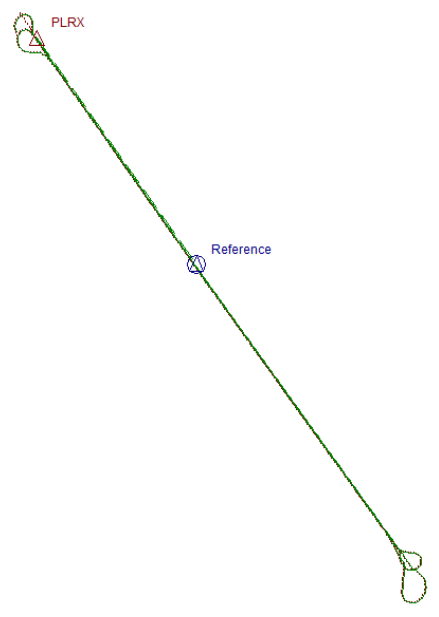

Fig. 1 Trajectory of flight

Table 3 summarizes the precision obtained from the PPP solution in terms of the average and maximum values of the standard deviation (STD) for the whole data set for the unknowns: point latitude, longitude, ellipsoidal height, receiver clock error, and Zenith dry+wet (Total) troposphere Delay (ZTD). The coordinates were computed in the ITRF2005 at the date of the 
GPS observation epoch. As the table shows, the average values of standard deviations for the latitude and longitude were at a few centimetres, and as expected, the height precision was almost double this value, but still at the sub-decimetre level. For most aerial mapping applications such precision is acceptable. The table also shows that maximum values of the standard deviations can reach a couple of decimetres. Figures 2 to 6 show the time series of the trend line of the computed STD (denoted as $\sigma$ ) of the PPP computed unknowns. The STD values were somewhat varying around the trend lines, thus, the trend line give a representative visual summary. Figure 7 illustrates the time series of the PPP computed ZTD, which shows that the solution has been stabilized after approximately 16 minutes.

Table 3 STD of the PPP solution for the unknowns

\begin{tabular}{|c|c|c|c|c|c|}
\hline \hline & $\sigma$ LAT $(\mathrm{m})$ & $\sigma \mathrm{LON}(\mathrm{m})$ & $\sigma \mathrm{HGT}(\mathrm{m})$ & $\sigma \mathrm{CLK}(\mathrm{ns})$ & $\sigma \mathrm{TZD}(\mathrm{m})$ \\
\hline Average & 0.044 & 0.028 & 0.0668 & 0.1614 & 0.003 \\
\hline Maximum & 0.227 & 0.176 & 0.425 & 0.584 & 0.005 \\
\hline
\end{tabular}

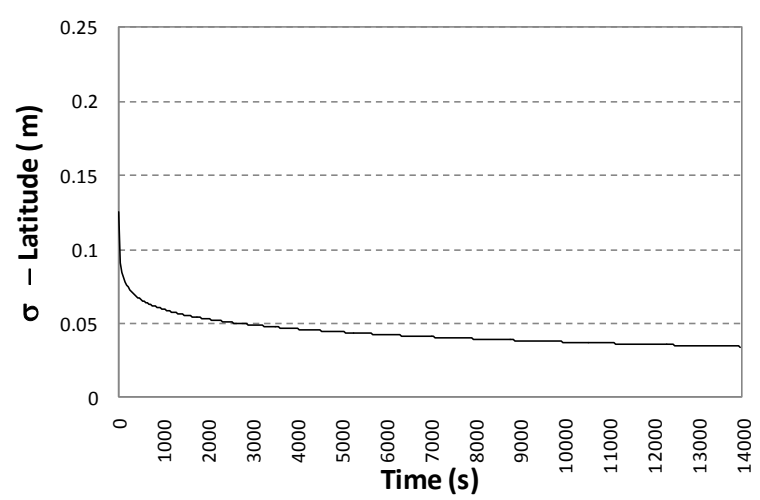

Fig. 2 Trend line of latitude STD

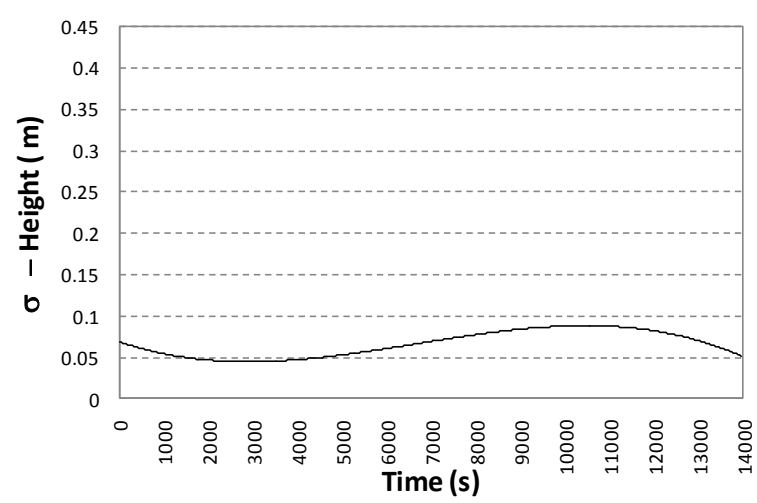

Fig. 4 Trend line of ellipsoidal height STD

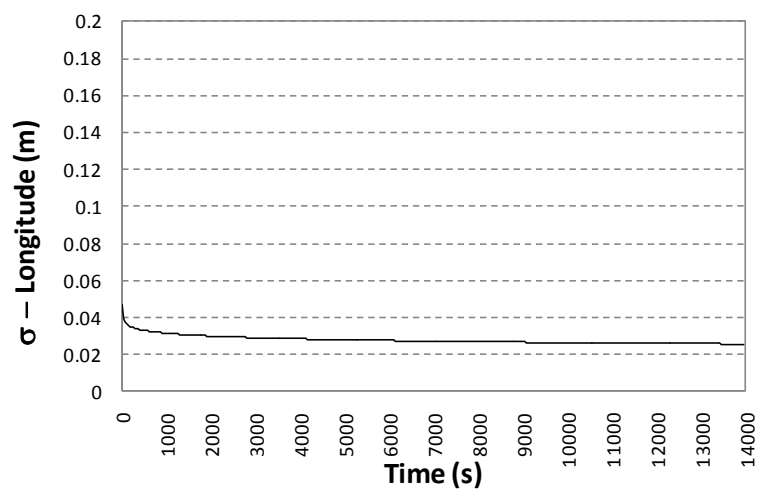

Fig. 3 Trend line of longitude STD

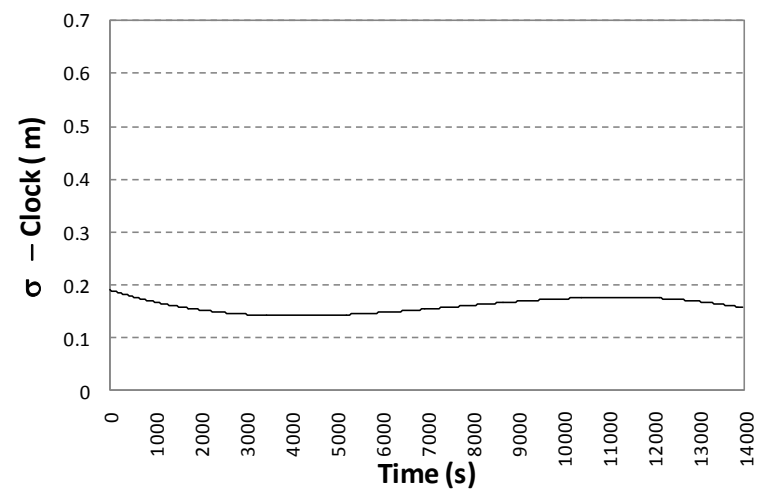

Fig. 5 Trend line of receiver clock STD 


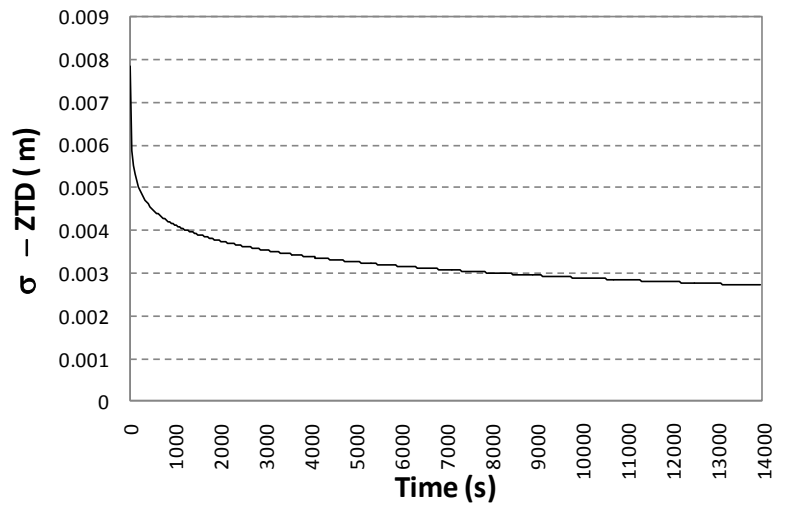

Fig. 6 Trend line of ZTD, STD

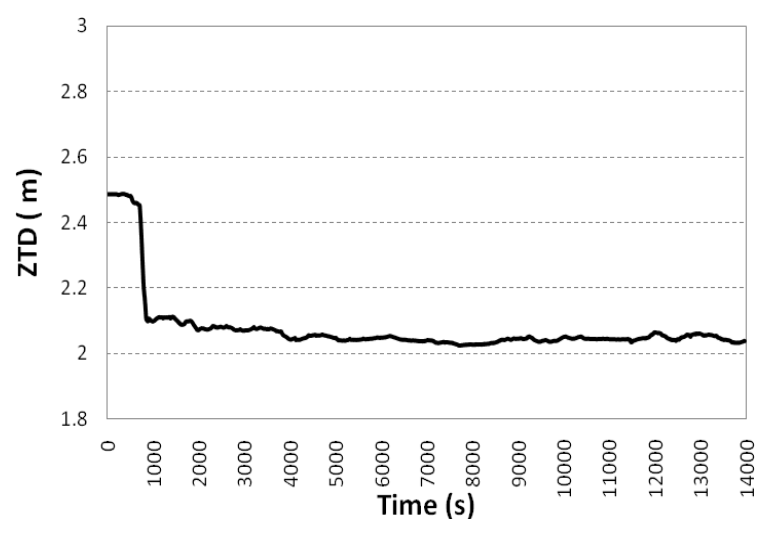

Fig. 7 Computed ZTD

For accuracy assessment of the PPP, the post-mission differential processing of data, used as the external reference, was performed using commercial software (Sokkia Spectrum, version 4.22). Due to flight manoeuvre, the data were lost at a few epochs, and at some other epochs the data of L2 was not recorded. Since these are exceptional circumstances, the comparison between the PPP and the differential solution was made for the period from 10:44:18 to 13:16:56, which included 9,145 epochs of data that include the enroute flying phase. Results of 10 epochs of anomalous differences were excluded from the comparison where the differences reached several metres, which can be attributed to observing only 4 satellites of low geometry, resulting in wrong ambiguity determination in the PPP solution. The fixedambiguity differential solution gave excellent standard deviations of $0.018 \mathrm{~m}, 0.009 \mathrm{~m}$, and $0.026 \mathrm{~m}$, on average, for the whole test period for latitude, longitude and height components, respectively. This justifies the use of the differential positioning scheme as the reference for comparison of the accuracy obtained from the PPP solution.

For the airborne test at hand, the differences between PPP and the differential solutions for the coordinate components are given in Table 4 and their time series are plotted in the Figures 8 , 9 and 10. As the table and the figures show, the differences were at the decimetre level for the latitude and the $\mathrm{cm}$ level for the longitude. This can be partially explained by the good distribution of the GPS satellites along the longitude direction compared with their distribution along the latitude direction. However, this should not give such a big ratio in discrepancy seen between latitude and longitude differences. Figure 8 shows that there could be a shift in latitude results of approximately $0.13 \mathrm{~m}$, which if applied would improve the overall average difference to $0.035 \mathrm{~m}$. This shift can be due to a shift of the coordinates of the reference station used in differential positioning or wrong ambiguity convergence in the PPP in the order of one cycle. The differences of the ellipsoidal heights were at 1.5 decimetre, on average. The change in differences in the middle of the trajectory can be attributed to a loss of observations for a period of 13 seconds, which requires re-initialization of the ambiguities. In this case, no backward positioning after solving for the ambiguities in the PPP solution was applied to examine result differences without backward processing. As shown from the figures, the period involved convergence of the PPP ambiguities suffered from positioning inaccuracy at 2-2.5 decimetres for latitude, and up to $1 \mathrm{~m}$ in height. 
Table 4 Differences between PPP and differential positioning results

\begin{tabular}{|l|l|l|l|}
\hline & \multicolumn{1}{|c|}{ LAT(m) } & LON(m) & HGT(m) \\
\hline Average & 0.134 & 0.007 & -0.168 \\
\hline $\begin{array}{l}\text { Maximu } \\
\text { m }\end{array}$ & 0.513 & 0.040 & 0.640 \\
\hline STD & 0.161 & 0.018 & 0.373 \\
\hline
\end{tabular}

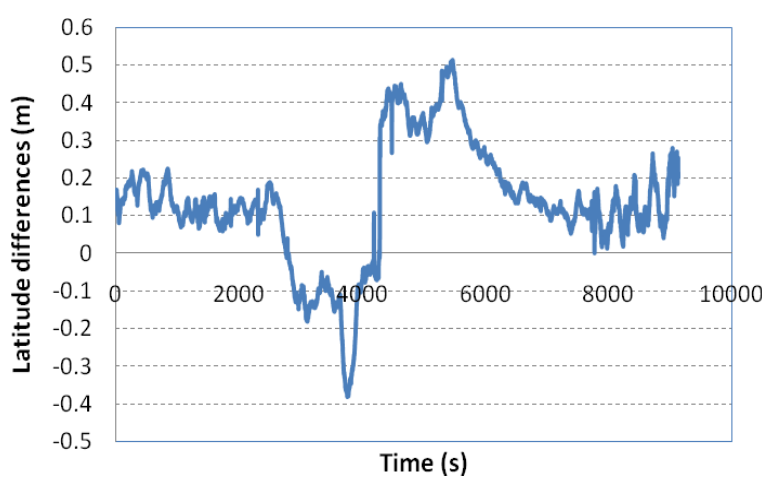

Fig. 8 PPP-Diff latitude differences

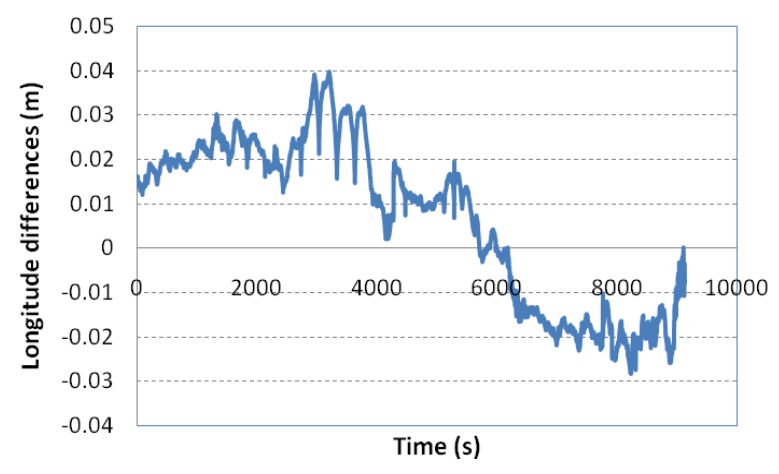

Fig. 9 PPP-Diff longitude differences

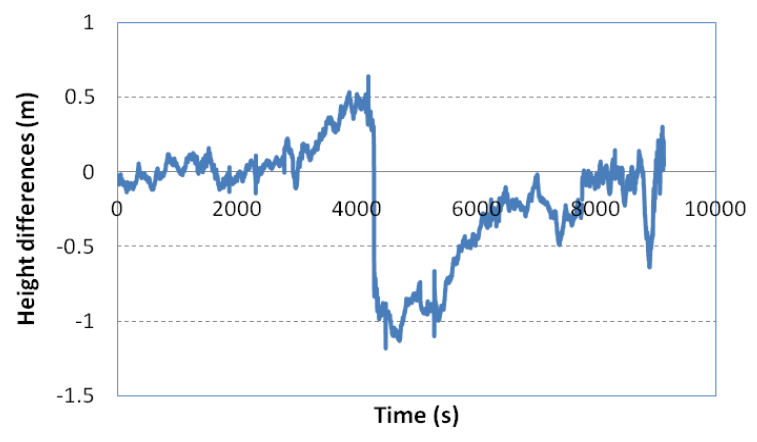

Fig. 10 PPP-Diff height differences

\section{Conclusions}

The precise Point Positioning approach is an un-differenced technique that can process the data in either post mission or real time. It has a particular advantage for airborne positioning that it is not dependent on ground reference stations that need to be located within a short distance. The paper shows that the PPP method can be successfully applied for the airborne mode. For the airborne test given in the paper, the PPP determined position components were precise at a few centimetres and it was double this value for height determination. The accuracy of PPP was within a few millimetres to 1.5 decimetre from a differential solution that was based on a fixed ambiguity resolution approach. This result was achieved with a sufficient data length that allows the float ambiguities computed in the PPP to converge to stable values. 


\section{Acknowledgment}

The Mathematical Geodesy \& Positioning group, the Delft Institute of Earth Observation and Space Systems, Delft University of Technology, Netherlands, is acknowledged for providing the airborne data used.

\section{References}

[1] Pedreira, P., "Optimistic Outlook for Galileo", GIM International, 2009, pp. 6-13

[2] Radišić, T., D. Novak, and T. Bucak "The Effect of Terrain Mask on RAIM Availability", The Journal of Navigation, 63-1, 2010, pp. 105-117.

[3] El-Mowafy, A. "Improving the Performance of RTK-GPS Reference Networks for Positioning in the Airborne Mode", Navigation, Journal of the American Institute of Navigation (ION), Vol (55), No.3, 2008, pp. 215-224.

[4] Kouba, J., and Héroux, P., "Precise Point Positioning Using IGS orbit and Clock Products", GPS Solutions, 5(2), 2001, pp. 12-28.

[5] Ge, M., Gendt, G., Rothacher, M., Shi, C., Geng, J., and Liu, J., "Resolution of GPS carrier-phase ambiguities in PPP with daily observations", J. Geodesy, 82(7), 2008, pp. 389-399.

[6] Shen, X. "Improving Ambiguity convergence in Carrier Phase-Based Precise Point Positioning”, UCGE Report No. 20170, Dept. of Geomatics Engineering, University of Calgary, Canada, 2002.

[7] El-Mowafy, A., "Precise Point Positioning for Mobile Mapping", Proc. of the 5th International Symposium on Mobile Mapping Technology MMT'07, Padua, Italy, May 28-31, 2007.

[8] Gao, Y. "Precise Point Positioning and Its Challenges, Aided-GNSS and Signal Tracking", Inside GNSS, 1(8), 2006, pp. 16-18.

[9] Abdel-Salam, M., "Precise Point Positioning Using Un-Differenced Code and Carrier Phase Observations", UCGE Report No. 20229, Dept. of Geomatics Engineering, The University of Calgary, Canada, 2005, $206 \mathrm{pp}$.

[10] Lahaye, F., Mireault, Y., Héroux, P., Tétreault, P. and Kouba, J., "A New, Timely Service from Natural Resources Canada", GPS World, Sept. 2008, accessed in Nov. 2010 online: http://sidt.gpsworld.com/gpssidt/article/articleDetail.jsp?id=549570.

[11] Altamimi, Z., Collilieux, X., Legrand, J. and Garayt, B., "ITRF2005: A new release of the International Terrestrial Reference Frame based on time series of station positions and Earth Orientation Parameters", J. Geophysical Research, B- Solid Earth, 112(B9): 2007, B09401.

[12] El-Mowafy, A., "An Alternative Post-Processing Positioning Approach Based on Precise Point Positioning with Corrections from a Reference Station", J. of Surveying Engineering, 135(2), 2009, pp. 56-65. 